\title{
Brain Neural Activity Patterns in an Animal Model of Antidepressant-Induced Manic Episodes
}

\section{OPEN ACCESS}

Edited by:

Nuno Sousa

University of Minho, Portugal

Reviewed by:

Gianluca Serafini,

San Martino Hospital (IRCCS), Italy

Eric C. Dumont,

Queen's University, Canada

${ }^{*}$ Correspondence:

Chuanjun Zhuo

chuanjunzhuotjmh@163.com:

chuanjunzhuo@nankai.edu.cn

Weihua Yue

dryue@bjmu.edu.cn

Xueqin Song

fccsongxq@zzu.edu.cn

${ }^{\dagger}$ These authors have contributed equally to this work

Specialty section:

This article was submitted to Emotion Regulation and Processing,

a section of the journal

Frontiers in Behavioral Neuroscience

Received: 09 September 2021

Accepted: 31 December 2021

Published: 16 February 2022

Citation:

Chen M, Chen G, Tian H, Dou G, Fang $T$, Cai Z, Cheng $L$, Chen $S$,

Chen C, Ping J, Lin X, Chen C,

Zhu J, Zhao F, Liu C, Yue W, Song $X$ and Zhuo $C$ (2022) Brain Neural

Activity Patterns in an Animal Model of Antidepressant-Induced Manic

Episodes.

Front. Behav. Neurosci. 15:771975. doi: 10.3389/fnbeh.2021.771975

\author{
Min Chen ${ }^{1 \dagger}$, Guangdong Chen ${ }^{2,3,4 t}$, Hongjun Tian ${ }^{5 t}$, Guangqian Dou ${ }^{5}$, Tao Fang ${ }^{5}$, \\ Ziyao Cai ${ }^{4}$, Langlang Cheng ${ }^{4}$, Suling Chen ${ }^{4}$, Ce Chen ${ }^{4}$, Jing Ping ${ }^{4}$, Xiaodong Lin ${ }^{4}$, \\ Chunmian Chen ${ }^{4}$, Jingjing Zhu ${ }^{4}$, Feifei Zhao ${ }^{4}$, Chuanxin Liu ${ }^{1}$, Weihua Yue ${ }^{6 *}$, \\ Xueqin Song ${ }^{7 *}$ and Chuanjun Zhuo ${ }^{5,7 *}$
}

\begin{abstract}
'Micro-imaging Center of Psychiatric Disorder, Institute of Mental Health, Jining Medical University, Jining, China, ${ }^{2}$ Center of Psychiatric Animal Model, Institute of Mental Health, Wenzhou Seventh Peoples Hospital, Wenzhou, China, ${ }^{3}$ Department of Psychiatry Medical Center, Wenzhou Seventh Peoples Hospital, Wenzhou, China, ${ }^{4}$ Department of Clinical Laboratory, Wenzhou Seventh Peoples Hospital, Wenzhou, China, ${ }^{5}$ Key Laboratory of Real Time Tracing of Brain Circuits in Psychiatry and Neurology (RTBNP_Lab), Nankai University Affiliated Tianjin Fourth Center Hospital, Tianjin Fourth Center Hospital, Tianjin, China, ${ }^{6}$ PKU-IDG/McGovern Institute for Brain Research, Peking University, Beijing, China, ${ }^{7}$ Department of Psychiatry, The First Affiliated Hospital of Zhengzhou University, Zhengzhou, China
\end{abstract}

Background: In the treatment of patients with bipolar disorder (BP), antidepressantinduced mania is usually observed. The rate of phase switching (from depressive to manic) in these patients exceeds $22 \%$. The exploration of brain activity patterns during an antidepressant-induced manic phase may aid the development of strategies to reduce the phase-switching rate. The use of a murine model to explore brain activity patterns in depressive and manic phases can help us to understandthe pathological features of BP. The novel object recognition preference ratio is used to assess cognitive ability in such models.

Objective: To investigate brain $\mathrm{Ca}^{2+}$ activity and behavioral expression in the depressive and manic phases in the same murine model, to aid understanding of brain activity patterns in phase switching in BP.

Methods: In vivo two-photon imaging was used to observe brain activity alterations in a murine model in which induce depressive-like and manic-like behaviors were induced sequentially. The immobility time was used to assess depressive-like symptoms and the total distance traveled was used to assess manic-like symptoms.

Results: In vivo two-photon imaging revealed significantly reduced brain $\mathrm{Ca}^{2+}$ activity in temporal cortex pyramidal neurons in the depressive phase in mice exposed to

Abbreviations: BD, Bipolar disorder; CUMS, Chronic unpredictable mild stress; EEG, Electroencephalographic; MRI, Magnetic resonance imaging; PPI, Prepulse inhibition; PP, Prepulse; PA, Startle stimulus; ReHo, Regional homogeneity; TPC, Temporal cortex. 
chronic unpredictable mild stress compared with naïve controls. The brain $\mathrm{Ca}^{2+}$ activity correlated negatively with the novel object recognition preference ratio within the immobility time. Significantly increased brain $\mathrm{Ca}^{2+}$ activity was observed in the ketamineinduced manic phase. However, this activity did not correlate with the total distance traveled. The novel object recognition preference ratio correlated negatively with the total distance traveled in the manic phase.

Keywords: bipolar disorder, brain calcium activity, phase switching, pre-pulse inhibition, symptoms' expression

\section{INTRODUCTION}

Bipolar disorder (BP) is a chronic psychiatric disorder affecting more than $1-4 \%$ of the global population (Grande et al., 2016). Although its clinical expression is usually associated with the disturbance of brain activity, the brain functional alterations occurring during depressive and manic episodes in patients with BP have not been described precisely. In addition, antidepressant use may induce manic episodes in at least $20 \%$ of patients with BP. Hypotheses regarding specific disturbances of brain neural activity, circuits, and networks in patients with BP have been supported by macro-neuroimaging studies, especially those in which functional magnetic resonance imaging (MRI) and electroencephalography (EEG) have been used (Berk, 2009; Berk et al., 2011, 2014; Fries et al., 2012; Schneider et al., 2012). Unexpectedly, these studies have demonstrated that brain alterations and cognitive impairment are consistently associated not with the timing of first BP episodes (whether depressive or manic), but with repeated manic or depressive episodes (El-Badri et al., 2001; Strakowski et al., 2002; Lyoo et al., 2006; Robinson and Ferrier, 2006).

Due to the limitations of conditions and lack of reference knowledge, the trajectories of dynamic alterations in brain functional activity, cognition, and behavior have rarely been observed in the same patient or animal model. The exploration of brain activity alterations in the same animal model during first the depressive phase and then the manic phase can provide evidence aiding the understanding of the pathological features of BP. Such models are particularly suitable for the examination of brain activity features in the antidepressant-induced manic phase of BP, as they often involve the use of ketamine to induce mania (Liu et al., 2018; Bhatt et al., 2021; Gao et al., 2021). Ketamine has an antidepressant effect and can rapidly alleviate serious depressive symptoms (Meshkat et al., 2021; Muscat et al., 2021).

In this pilot study, we used in vivo two-photon imaging to examine brain activity features in the depressive and manic phases in the same murine model of BP. As depressive episodes usually precede manic episodes in clinical practice, we modeled depressive episodes using chronic unpredictable mild stress (CUMS), followed by the induction of the manic phase with ketamine (thus representing antidepressant-induced mania in BP) in the same animals. We proposed two hypotheses: (1) that brain $\mathrm{Ca}^{2+}$ activity patterns in the depressive and manic phases would differ, and (2) that brain $\mathrm{Ca}^{2+}$ alterations would be associated with cognitive and behavioral performance.

\section{MATERIALS AND METHODS}

\section{Animals and Experimental Design}

Male C57BL/6 mice from multiple litters (Japan SLC Inc., Shizuoka, Japan) were used in this study. Eight groups of six mice each were housed in group cages. The experiments were performed using polycarbonate cages $(18 \times 30 \times 17 \mathrm{~cm})$ designed for " $24 \mathrm{~h}$ home-cage activity and social behavior monitoring" (Ohara Co. Ltd., Tokyo, Japan). Four mice were kept in each cage. The mice were supplied with Palsoft paper bedding (Oriental Yeast, Tokyo, Japan). The animal room was maintained at $50 \% \pm 10 \%$ humidity and a temperature of $23 \pm 2{ }^{\circ} \mathrm{C}$ under a 12 -h light/12-h dark cycle (lights on from 06:00-18:00). The mice were allowed free access to CE-2 food (CLEA Japan, Tokyo, Japan) and water. The mice were maintained and all experiments were performed in accordance with guidelines provided by National Institute of Mental Health ${ }^{1}$, and all procedures were approved by the Committee for Animal Care and Use of Tianjin Medical University Affiliated Tianjin Fourth Centre Hospital (IRB-no. 801758).

First, we established the depressive phase by exposing the mice to CUMS using a standard protocol to provoke depressive behavior. One day later, according to a $\mathrm{BP}$ model designed to mimic a protocol for mania prevention (Banwari et al., 2015; Bhatt et al., 2021; Gao et al., 2021), we initiated a course of daily intraperitoneal injection of ketamine $(25 \mathrm{mg} / \mathrm{kg})$ to provoke manic behavior (Liu et al., 2018; Bhatt et al., 2021; Gao et al., 2021). Assessments were performed after the establishment of each phase.

\section{Stereotaxic Injection}

Anesthesia was induced with $1.25 \%$ avertin, the scalp was incised and locally sterilized, and the periosteal tissue was removed. A stereotaxic instrument (RWD, China) was used to identify the hindlimb region of the primary somatosensory cortex (S1HL; about $0.5 \mathrm{~mm}$ anterior to and $1.5 \mathrm{~mm}$ lateral of bregma). An injection hole was created on the cranium with a high-speed microdrill (OmniDrill35; WPI, USA), and a glass microelectrode connected to an ultra-micro-injection pump (Nanoliter 2010; WPI) was used to inject $80 \mathrm{nl}$ AAV2/1-hSyn-GCaMP6 s or $150 \mathrm{nl}$ AAV2/1-hSyn-DIO-GCaMP6s (>1 × $10^{13}$ gene copies/ml; University of Pennsylvania Gene Therapy Program Vector Core) into the fifth cortical layer at a $60^{\circ}$ angle to avoid imaging site

\footnotetext{
${ }^{1}$ https://www.nih.gov/about-nih/what-we-do/nih-almanac/national-institutemental-health-nimh
} 
damage. The glass electrode was kept in the brain tissue for a total of $5 \mathrm{~min}$.

\section{In vivo Two-Photon Calcium Imaging}

Three weeks after stereotaxic injection, the mice were anesthetized with $1.25 \%$ avertin, the skull was exposed, and two metal bars were attached to the rostral and caudal portions of the skull, respectively, with glue (Loctite 401) and dental cement to ensure head restraint during imaging. One day later, a high-speed microdrill was used to create an imaging window above S1HL. A glass coverslip was applied to the window using Vetbond tissue adhesive (3M, USA).

In vivo calcium imaging was performed on awake mice under head restraint using a 920-nm excitation laser with a water-immersed objective $(20 \times, 1.1$ numerical aperture; Zeiss, Germany). Under an LSM780 two-photon microscope (Zeiss), calcium activity was recorded at $2 \mathrm{~Hz}$ for $2.5 \mathrm{~min}$ at the apical tufts $(0-80 \mu \mathrm{m}$ from pia), vasoactive intestinal polypeptide somas and axons (200-300 $\mu \mathrm{m}$ from pia), somatostatin somas and axons (400-500 $\mu \mathrm{m}$ from pia), and layer 5 pyramidal neuron somas (600-650 $\mu \mathrm{m}$ from pia). These regions of interest were defined manually. Calcium-signal time series were corrected using the TurboReg plugin for ImageJ software (National Institutes of Health, Bethesda, MD, USA). Mean pixel values were averaged to obtain fluorescent $(F)$ values, normalized as $\left(\Delta F-F_{0}\right) / F_{0}$, where $F_{0}$ (the baseline value) was the average obtained during the first $10 \%$ of recording. Total integrated calcium values were calculated by summing $\Delta F / F_{0}$ for the entire time series. Calcium spikes were defined as $\geq 3$-standarddeviation increases.

\section{Behavioral Assays}

The animals were subjected to a sucrose preference test as described previously (Can et al., 2012; Sharma et al., 2016), followed by a prepulse inhibition (PPI) test adapted for the quantification of sensory gating function (Feldcamp et al., 2017). After acclimation of the mice in a sound-isolating chamber with $65 \mathrm{~dB}$ background noise, a 75- $\mathrm{dB}$ prepulse (PP) was applied for $20 \mathrm{~ms}$, followed $100 \mathrm{~ms}$ later by a $40-\mathrm{ms} 120$-dB startle stimulus (PA). The mice completed three trials with intervening intervals of $30 \mathrm{~s}$. Scores were averaged and the PPI ratio was calculated as $(\mathrm{PA}-\mathrm{PP}) / \mathrm{PA} \times 100 \%$.

\section{Statistical Analysis}

Data are presented as means \pm standard errors of the mean unless specified otherwise. Data were compared using a one-way analysis of variance and post hoc Tukey tests. Data analysis and figure plotting were performed with the GraphPad Prism software (version 8.0).

\section{RESULTS}

\section{CUMS and Ketamine Exposure Evoked Abnormal Cortical Transmission and Behavior}

Compared with naïve controls, mice exposed to CUMS had significantly longer immobility times in the depressive phase
$(P<0.001$; Figure 1C) and significantly longer total distances traveled in the manic phase $(P<0.001$; Figure $2 \mathrm{C})$. Two-photon calcium imaging demonstrated significantly decreased $\mathrm{Ca}^{2+}$ activity in temporal cortex (TPC) pyramidal neurons in the depressive phase, and significantly increased activity in the manic phase (Figures 1A,B, 2A,B). Compared with the naive controls, $\mathrm{Ca}^{2+}$ hypoactivity was observed in the prefrontal cortex in the depressive and manic phases in mice exposed to CUMS followed by ketamine (Figures 1E,F, 2E,F). The normalized total integrated calcium values correlated significantly with immobility times in the depressive phase, but not the manic phase (both $P<0.001$; Figures 1D, 2B), due primarily to decreased calcium spike frequencies $(P<0.001$; Figures 2 C,D). In both phases, exposed mice had poorer novel object recognition preference ratios than did controls (both $P<0.001$; Figures 1G, $2 \mathrm{G})$. This ratio correlated negatively with the immobility time in the depressive phase and with the total distance traveled in the manic phase (Figures $\mathbf{1 H}, \mathbf{2} \mathbf{H}$ ). Thus, mice exposed to CUMS and ketamine showed dual phenotypes of depressive and manic behavior largely consistent with the behavioral expression of patients with $\mathrm{BP}$, reflecting the successful establishment of the BP model with an antidepressant-induced manic phase. They exhibited impaired cognitive ability in the depressive and manic phases, which was more severe in the manic phase.

\section{Relationships Among Brain $\mathrm{Ca}^{2+}$ Activity, Symptom Severity, and Cognitive Impairment}

Brain $\mathrm{Ca}^{2+}$ activity correlated negatively with the severity of depressive symptoms (as reflected by the immobility time; Figure 1D). A similar, but nonsignificant trend was observed for the severity of manic behavior (Figure 2D). Cognitive impairment was observed in the depressive and manic phases but was more severe in the manic phase.

\section{DISCUSSION}

This pilot microimaging study conducted with a murine model of BP with an antidepressant-induced manic phase yielded three main findings. First, compared with naïve controls, exposed mice showed significantly decreased brain $\mathrm{Ca}^{2+}$ activity in the depressive phase, which is consistent with macro-imaging findings suggesting that the disruption of the emotional network contributes to mood dysregulation in patients with BP (Drevets et al., 1997; Kempton et al., 2008; Bora et al., 2010; EllisonWright and Bullmore, 2010; Nortje et al., 2013; Hibar et al., 2018; Fries et al., 2019; Matsuo et al., 2019; Harrison et al., 2021). Second, brain $\mathrm{Ca}^{2+}$ activity did not increase in the manic phase, inconsistent with some previous findings (Ghedim et al., 2012; Ettenberg et al., 2020; Gao et al., 2021). This difference could be explained by the design of our murine model, in which the mice received a "double hit" due to the use of CUMS followed 1 day later by ketamine, in contrast to models of ketamine-induced manic behavior alone. The mice may not have had sufficient time to recover from the brain alterations caused by CUMS before ketamine injection, leading to the lack of an increase in brain 
A
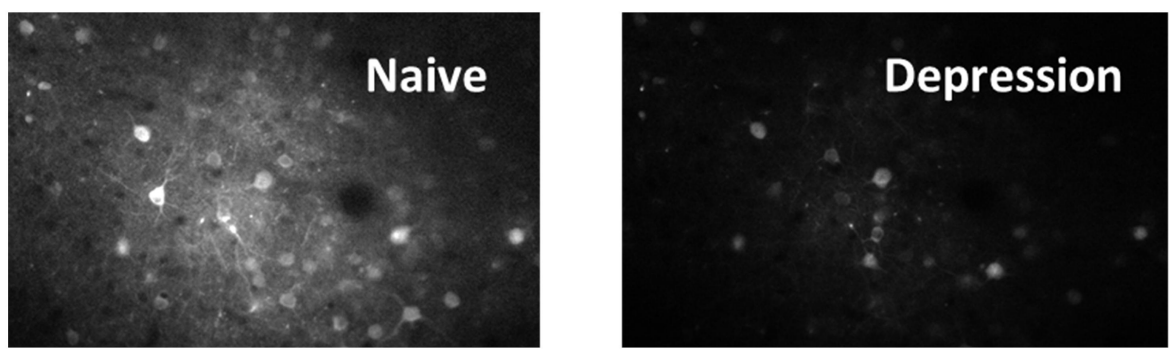

B

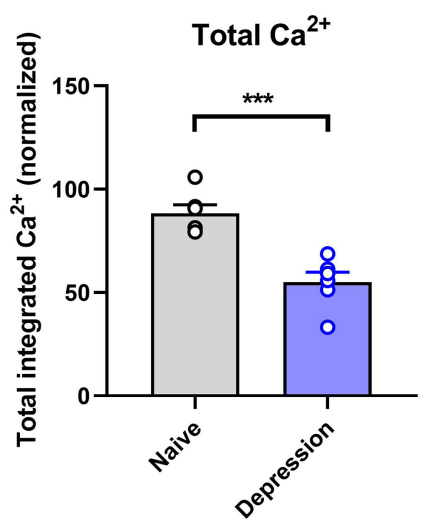

C

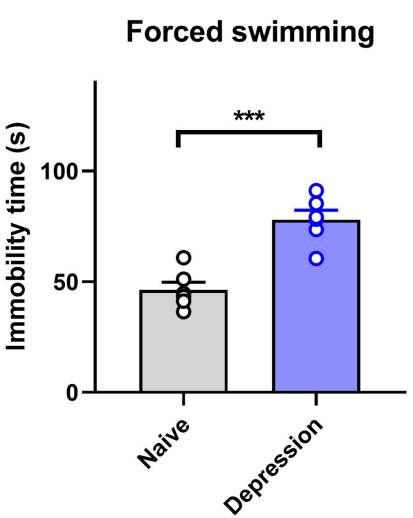

D

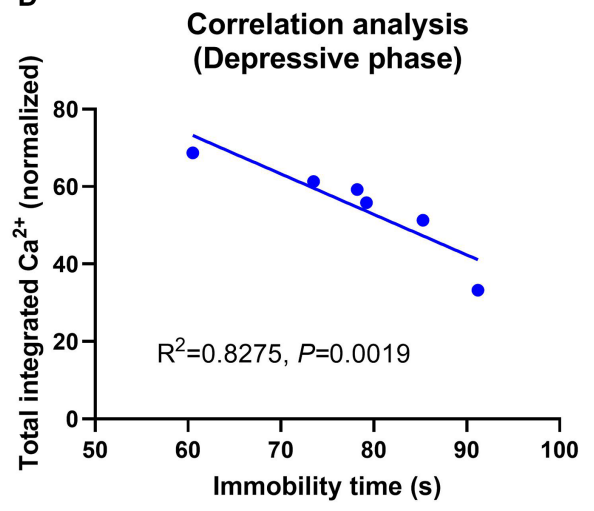

E
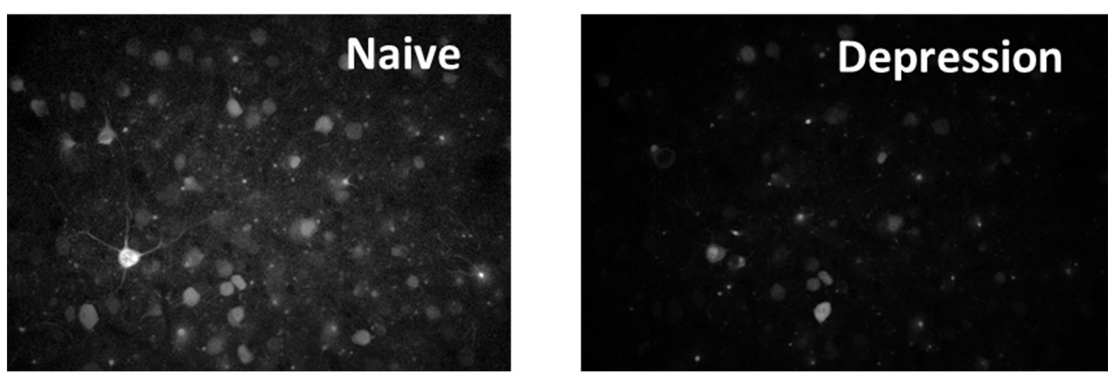

$\mathbf{F}$

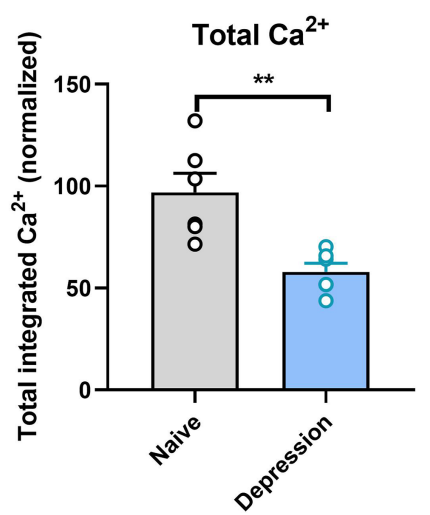

G

Novel object recognition

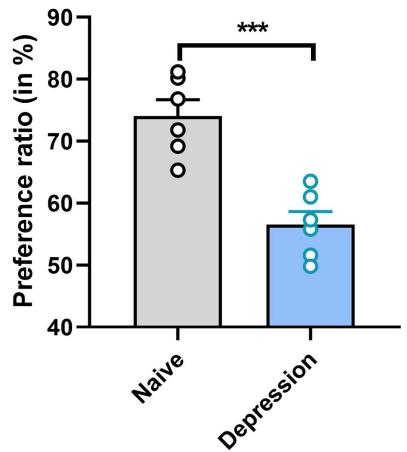

H

Correlation analysis

(Depressive phase)

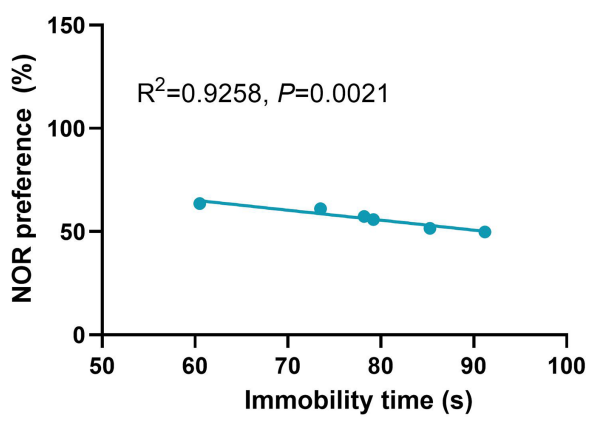

FIGURE 1 | Brain $\mathrm{Ca}^{2+}$ activity and behavioral index alterations in mice with BPD exposed to CUMS and controls. Note: Compared with the naive group (six mice, group-housed), mice in the depressive phase ( $n=6$, group-housed) showed significantly decreased normalized integrated $\mathrm{Ca}^{2+}$ activity $(96.28 \pm 11.17 \mathrm{vs}$. $53.00 \pm 8.25, P<0.001, \mathbf{A}, \mathbf{B})$ and more immobility time (86.52 \pm 9.22 vs. $47.67 \pm 5.31, \mathbf{C})$, and these variables correlated with each other (D); mice in the depressive phase also showed $\mathrm{Ca}^{2+}$ hypoactivity in the prefrontal cortex $(\mathbf{E}, \mathbf{F})$, and had poorer novel object recognition preference ratios $(\mathbf{G})$. The preference ratio (pre-pause index) also correlated with the immobility time $\mathbf{( H ) .}{ }^{* *} P<0.01 ;{ }^{* \star *} P<0.001$, vs. the naiive group. 
A

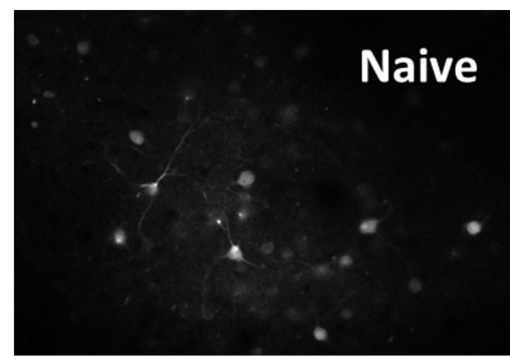

B

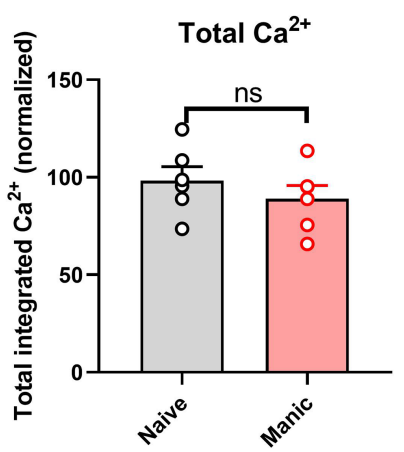

C

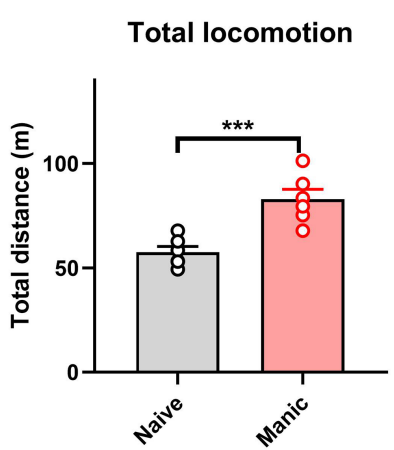

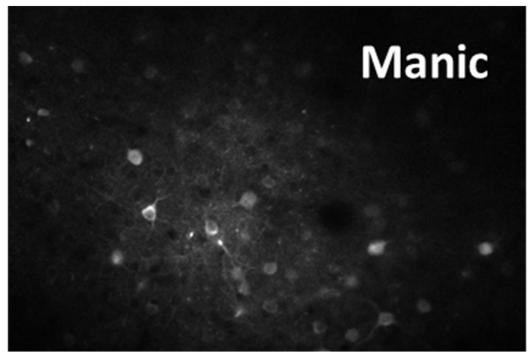

D

E
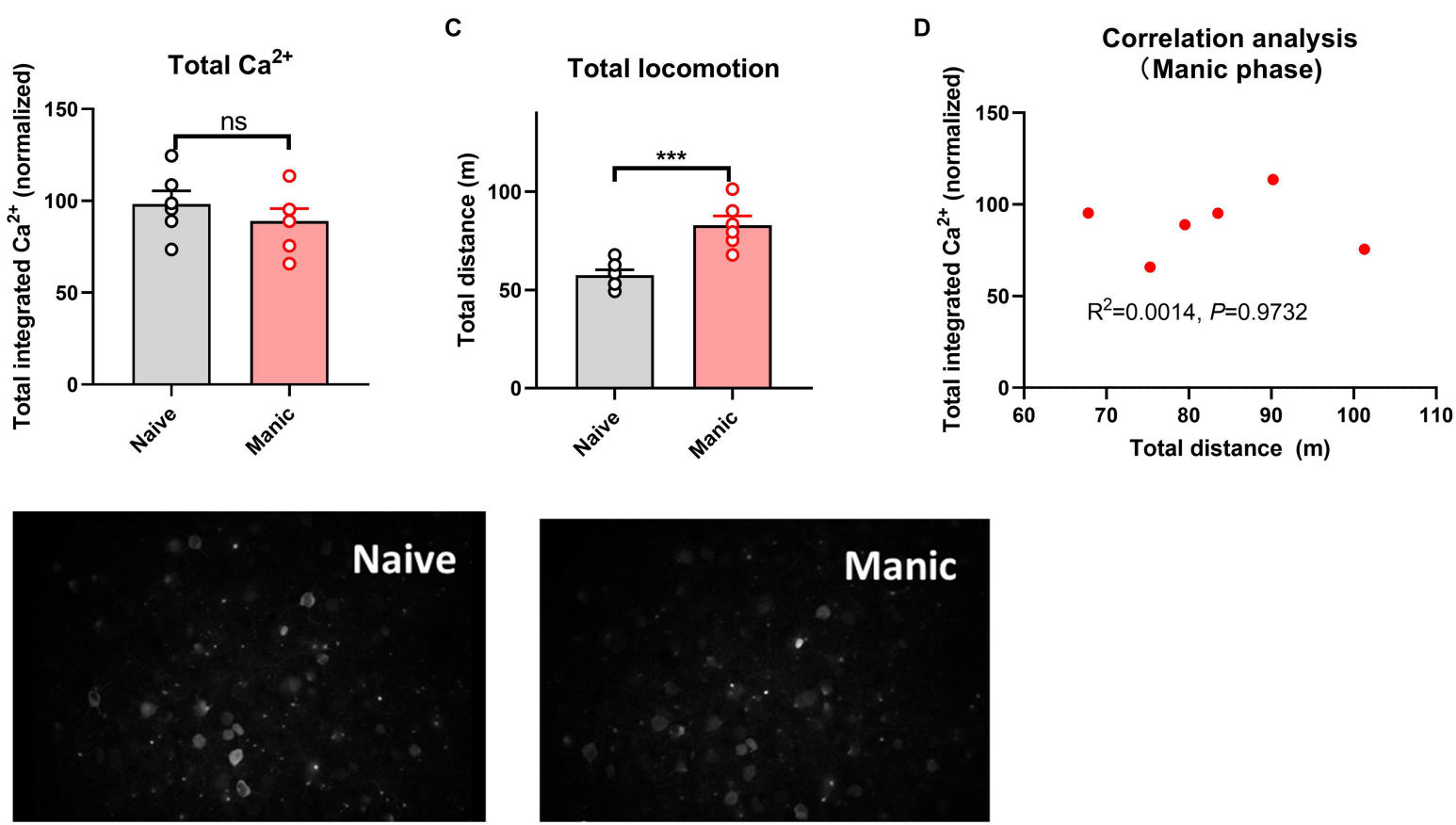

orrelation analysis

(Manic phase)

Total distance $(\mathrm{m})$

F

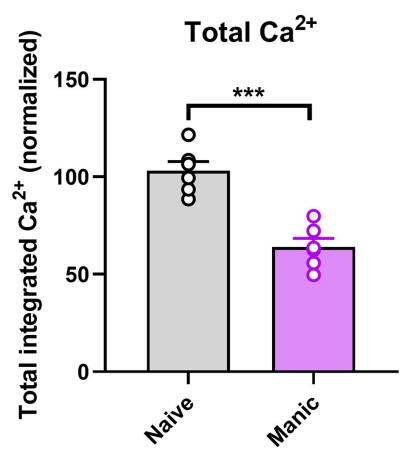

G

Novel object recognition

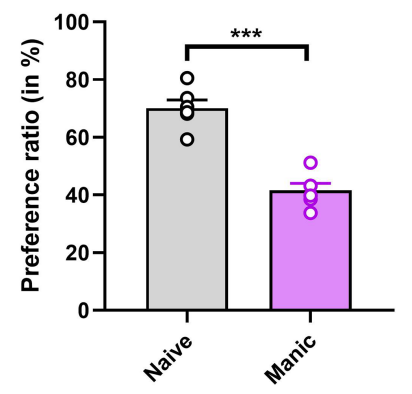

H

\section{Correlation analysis \\ (Manic phase)}

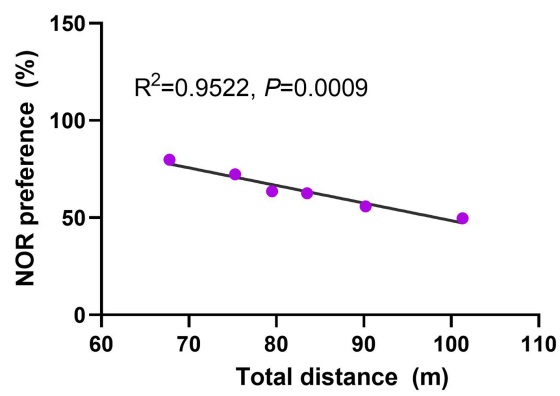

FIGURE 2 | Brain $\mathrm{Ca}^{2+}$ activity and behavioral index alterations in mice with BPD exposed to ketamine and controls. Note: Compared with the naïve group (six mice, group-housed), mice in the manic phase $\left(n=6\right.$, group-housed) showed similar normalized integrated $\mathrm{Ca}^{2+}$ activity $(96.89 \pm 13.00$ vs. $92.18 \pm 10.44, \mathbf{A}, \mathbf{B})$ but significantly more total locomotion $(53.11 \pm 9.25$ vs. $79.66 \pm 7.77 \mathrm{~m}, \mathbf{C})$; mice in the manic phase also showed Ca ${ }^{2+}$ hypoactivity in the prefrontal cortex (E,F), and had poorer novel object recognition preference ratios (G). The preference ratio, but not the normalized integrated Ca ${ }^{2+}$ activity, correlated with the total distance traveled (D, $\mathbf{H}) .{ }^{\star \star \star} P<0.001$, vs. the naïve group; ns, not significant.

$\mathrm{Ca}^{2+}$ activity in the manic phase. Third, cognitive impairment was a common feature of the depressive and manic phases and correlated with the severity of depressive and manic symptoms, but it was more severe in the manic phase.
Numerous reports, especially those on macro-imaging (particularly MRI) studies, describe decreased brain activity in the TPC in the depressive and manic phases of BP. For example, Cerullo et al. (2014) reported decreased middle 
temporal gyrus activation during the depressive phase in patients with BP and those with unipolar depression. Xiao et al. (2019) observed reduced cortical regional homogeneity (ReHo) in the superior temporal gyrus and increased ReHo in the cerebellum in the manic phase in patients with BP relative to a euthymic group. The Elderly Nutritional Index for Geriatric Malnutrition Assessment Bipolar Disorder Working Group also reported widespread bilateral patterns of reduced cortical thickness in the frontal, temporal, and parietal regions among 6,503 adults with BP (Hibar et al., 2018). These findings, along with our microimaging findings, converge to indicate that the ability to adjust the intrinsic emotional network is impaired in animal models of BP and in patients with this disorder.

Our finding that brain $\mathrm{Ca}^{2+}$ activity was related negatively only to the severity of depressive-like symptoms is supported by macro-imaging findings. For example, macro-imaging studies have documented correlations of depressive symptom severity with brain functional and structural alterations (Vai et al., 2019; Gong et al., 2020, 2021; Angelescu et al., 2021; Cahn et al., 2021; Keramatian et al., 2021). EEG studies have revealed correlations between brain neuron impairment and the severity of depressive symptoms (Banwari et al., 2015; Zhou et al., 2015; Liu et al., 2018; Belleau et al., 2019; Bhatt et al., 2021; Gao et al., 2021). We found no correlation between brain neuron impairment and the severity of manic-like symptoms, inconsistent with many previous findings (Dixon et al., 1994; Brody et al., 2003; Ettenberg et al., 2020; Gao et al., 2021), which may be related to the "double-hit" nature of our murine model. Further studies are needed to explore the precise pathological features of BP.

Clinical and macro-neuroimaging findings suggest that cognitive impairment is a core symptom in patients with BP; micro-neuroimaging studies have also revealed cognitive impairment in animal models of mania and depression (Banwari et al., 2015; Zhou et al., 2015; Hiser and Koenigs, 2018; Belleau et al., 2019; Mondimore, 2020; Harrison et al., 2021; Bhatt et al., 2021; Gao et al., 2021). Our data constitute new evidence for this phenomenon.

In summary, the use of in vivo two-photon imaging to examine a murine model of BP reveals brain neural activity patterns in the depressive and manic phases of this disorder (although the use of ketamine to induce the manic phase needs to be assessed further; the use of another method that does not cause brain neurophysiological alterations would be ideal). The consecutive induction of the depressive and manic phases in the same mice enables characterization of the trajectory of these patterns, providing information for the examination of phase switching in patients who experience antidepressant-induced mania. In turn, this characterization can form the basis for the examination of the effects of different treatment strategies in terms of improvement in brain activity and provide reference information for clinical practice. Although the translation of basic research findings to clinical practice is a lengthy process that is not guaranteed to succeed, translational research is needed to promote advances in therapeutics.

\section{Limitations}

This study has several limitations. First, the model used in this study and others, in which ketamine is used to induce the manic phase, is valid, but the accuracy of this assessment has not been discussed in past years, although the aims of previous studies were to explore new methods for the improvement of treatment effects in patients with treatment-resistant depression and to rapidly reduce suicide attempts due to severe depressive symptoms in BP (Valvassori et al., 2019; Kim and Monteggia, 2020; McIntyre et al., 2020; Chen et al., 2021; Dean et al., 2021; Keilp et al., 2021; Magalhães et al., 2021; Nikayin and Sanacora, 2021; Wilkowska et al., 2021). A reviewer of this article led us to rethink the validity of the use of ketamine to induce the manic phase when studying BP (a physiopathological process), as drug-induced manic behavior may have different underlying mechanisms, Strictly speaking, a ketamine-induced manic phase can only represent such a phase induced by antidepressants, which is usually observed in clinical practice. The use of ketamine (including es-ketamine and dextroamphetamine, previously shown to be inferior for the treatment of depression) to treat serious depression may be better than other approaches, but its use to model mania in studies of BP-related brain features requires further examination. Ketamine causes brain neurophysiological alterations leading to mania-like behavior, therefore, its use to explore treatments targeting depressive symptoms is suitable, but we believe that model improvement is needed for applications. In future research, multiple strategies should be explored to fully represent the manic phase of BP. Although the findings of the present study have limited value, they provide a basis for further study. Second, our findings may be attributable to our induction of the manic phase immediately after the depressive phase in this study. An intervening interval of time may have allowed for brain recovery, avoiding a "double hit" effect of the CUMS and ketamine treatments. However, this dual-modeling approach has not been attempted in previous studies, and experience from clinical practice suggests that most patients with BP switch phases rapidly; some patients even have mixed manic/depressive episodes (Sharma et al., 2016). In the future, we will design and conduct multiple-arm studies to clarify this issue and identify a better method for BP modeling. Third, we used only a few behavioral and cognitive indices, although these indices are classic for animal models. The development of additional tests for the assessment of cognitive alterations might aid a more detailed description of the pathological mechanisms of BP.

\section{CONCLUSION}

In this study, we observed brain $\mathrm{Ca}^{2+}$ activity in an animal model of BP with a chronic unpredictable mild stress-induced depressive phase and a subsequent antidepressant-induced manic phase. In the depressive phase, brain neural activity alterations correlated with depressive symptoms and cognitive impairment. In the manic phase, cognitive impairment, but not brain neural activity, correlated with manic symptoms. Findings from this animal study expand our understanding of phase- 
switching mechanisms in patients with BP, but further research is needed.

\section{DATA AVAILABILITY STATEMENT}

The datasets generated and analyzed during the present study are available from the corresponding author upon reasonable request.

\section{ETHICS STATEMENT}

The study was approved by the Committee for Animal Care and Use of Tianjin Medical University Affiliated Tianjin Fourth Centre Hospital (IRB-no. 801758).

\section{AUTHOR CONTRIBUTIONS}

$\mathrm{CZ}$ and HT conceived and designed the research. MC, GC, GD, TF, ZC, LC, SC, CeC, JP, XL, ChC, JZ, FZ, and CL collected

\section{REFERENCES}

Angelescu, I., Brugger, S. P., Borgan, F., Kaar, S. J., and Howes, O. D. (2021). The magnitude and variability of brain structural alterations in bipolar disorder: a double meta-analysis of 5534 patients and 6651 healthy controls. J. Affect. Disord. 291, 171-176. doi: 10.1016/j.jad.2021.04.090

Banwari, G., Desai, P., and Patidar, P. (2015). Ketamine-induced affective switch in a patient with treatment-resistant depression. Indian J. Pharmacol. 47, 454-455. doi: 10.4103/0253-7613.161277

Belleau, E. L., Treadway, M. T., and Pizzagalli, D. A. (2019). The impact of stress and major depressive disorder on hippocampal and medial prefrontal cortex morphology. Biol. Psychiatry 85, 443-453. doi: 10.1016/j.biopsych.2018.09.031

Berk, M. (2009). Neuroprogression: pathways to progressive brain changes in bipolar disorder. Int. J. Neuropsychopharmacol. 12, 441-445. doi: 10.1017/S1461145708009498

Berk, M., Berk, L., Dodd, S., Cotton, S., Macneil, C., Daglas, R., et al. (2014). Stage managing bipolar disorder. Bipolar. Disord. 16, 471-477. doi: 10.1111/bdi. 12099

Berk, M., Kapczinski, F., Andreazza, A. C., Dean, O. M., Giorlando, F., Maes, M., et al. (2011). Pathways underlying neuroprogression in bipolar disorder: focus on inflammation, oxidative stress and neurotrophic factors. Neurosci. Biobehav. Rev. 35, 804-817. doi: 10.1016/j.neubiorev.2010.10.001

Bhatt, K., Yoo, J., and Bridges, A. (2021). Ketamine-induced manic episode. Prim. Care Companion CNS Disord. 23:20102811. doi: 10.4088/PCC.20102811

Bora, E., Fornito, A., Yücel, M., and Pantelis, C. (2010). Voxelwise meta-analysis of gray matter abnormalities in bipolar disorder. Biol. Psychiatry 67, 1097-1105. doi: 10.1016/j.biopsych.2010.01.020

Brody, S. A., Geyer, M. A., and Large, C. H. (2003). Lamotrigine prevents ketamine but not amphetamine-induced deficits in prepulse inhibition in mice. Psychopharmacology (Berl) 169, 240-246. doi: 10.1007/s00213-0031421-2

Cahn, A. J., Keramatian, K., Frysch, C., Yatham, L. N., and Chakrabarty, T. (2021). Longitudinal grey matter changes following first episode mania in bipolar I disorder: a systematic review. J. Affect. Disord. 291, 198-208. doi: 10.1016/j.jad. 2021.04.051

Can, A., Dao, D. T., Arad, M., Terrillion, C. E., Piantadosi, S. C., and Gould, T. D. (2012). The mouse forced swim test. J. Vis. Exp. 59:e3638. doi: 10.3791/ 3638

Cerullo, M. A., Eliassen, J. C., Smith, C. T., Fleck, D. E., Nelson, E. B., Strawn, J. R., et al. (2014). Bipolar I disorder and major depressive disorder show similar brain activation during depression. Bipolar Disord. 16, 703-712. doi: $10.1111 /$ bdi. 12225 the data and conducted the research. MC, GC, HT, WY, XS, and $\mathrm{CZ}$ analyzed and interpreted the data. $\mathrm{CZ}$ and $\mathrm{HT}$ wrote the initial manuscript. WY and XS revised the manuscript. CZ had primary responsibility for the final manuscript content. All authors read and approved the final manuscript. All authors contributed to the article and approved the submitted version.

\section{FUNDING}

This work was supported by grants from the National Natural Science Foundation of China (81871052 and 82171053 to CZ); National Key R\&D Program of China (2016YFC1307000 to WY); the Key Projects of the Natural Science Foundation of Tianjin, China (17JCZDJC35700 to CZ); the Tianjin Health Bureau Foundation (2014KR02 to $\mathrm{CZ);}$ and the Tianjin Science and Technology Bureau (15JCYBJC50800 to HT).

Chen, M., Tian, H., Huang, G., Fang, T., Lin, X., Shan, J., et al. (2021). alcium imaging reveals depressive- and manic-phase-specific brain neural activity patterns in a murine model of bipolar disorder: a pilot study. Transl. Psychiatry 11:619. doi: 10.1038/s41398-021-01750-8

Dean, R. L., Marquardt, T., Hurducas, C., Spyridi, S., Barnes, A., Smith, R., et al. (2021). Ketamine and other glutamate receptor modulators for depression in adults with bipolar disorder. Cochrane Database Syst. Rev. 10:CD011611. doi: 10.1002/14651858.CD011611.pub3

Dixon, J. F., Los, G. V., and Hokin, L. E. (1994). Lithium stimulates glutamate "release" and inositol 1,4,5-trisphosphate accumulation via activation of the Nmethyl-D-aspartate receptor in monkey and mouse cerebral cortex slices. Proc. Natl. Acad. Sci. U S A 91, 8358-8362. doi: 10.1073/pnas.91.18.8358

Drevets, W. C., Price, J. L., Simpson, J. R., Jr., Todd, R. D., Reich, T., Vannier, M., et al. (1997). Subgenual prefrontal cortex abnormalities in mood disorders. Nature 386, 824-827. doi: 10.1038/386824a0

El-Badri, S. M., Ashton, C. H., Moore, P. B., Marsh, V. R., and Ferrier, I. N. (2001). Electrophysiological and cognitive function in young euthymic patients with bipolar affective disorder. Bipolar Disord. 3, 79-87. doi: 10.1034/j.1399-5618. 2001.030206.x

Ellison-Wright, I., and Bullmore, E. (2010). Anatomy of bipolar disorder and schizophrenia: a meta-analysis. Schizophr. Res. 117, 1-12. doi: 10.1016/j.schres. 2009.12.022

Ettenberg, A., Ayala, K., Krug, J. T., Collins, L., Mayes, M. S., and Fisher, M. P. A. (2020). Differential effects of lithium isotopes in a ketamine-induced hyperactivity model of mania. Pharmacol. Biochem. Behav. 190:172875. doi: $10.1016 /$ j.pbb.2020.172875

Feldcamp, L. A., Boutros, P. C., Raymond, R., Fletcher, P. J., Nobrega, J. N., and Wong, A. H. C. (2017). Pdxdc1 modulates prepulse inhibition of acoustic startle in the mouse. Transl. Psychiatry 7:e1125. doi: 10.1038/tp.2017.85

Fries, G. R., Lima, C. N. C., Valvassori, S. S., Zunta-Soares, G., Soares, J. C., and Quevedo, J. (2019). Preliminary investigation of peripheral extracellular vesicles' microRNAs in bipolar disorder. J. Affect. Disord. 255, 10-14. doi: 10.1016/j.jad.2019.05.020

Fries, G. R., Pfaffenseller, B., Stertz, L., Paz, A. V., Dargél, A. A., Kunz, M., et al. (2012). Staging and neuroprogression in bipolar disorder. Curr. Psychiatry Rep. 14, 667-675. doi: 10.1007/s11920-012-0319-2

Gao, T. H., Ni, R. J., Liu, S., Tian, Y., Wei, J., Zhao, L., et al. (2021). Chronic lithium exposure attenuates ketamine-induced mania-like behavior and c-Fos expression in the forebrain of mice. Pharmacol. Biochem. Behav. 202:173108. doi: $10.1016 /$ j.pbb.2021.173108

Ghedim, F. V., Fraga Dde, B., Deroza, P. F., Oliveira, M. B., Valvassori, S. S., Steckert, A. V., et al. (2012). Evaluation of behavioral and neurochemical 
changes induced by ketamine in rats: implications as an animal model of mania. J. Psychiatr. Res. 46, 1569-1575. doi: 10.1016/j.jpsychires.2012.08.010

Gong, J., Wang, J., Chen, P., Qi, Z., Luo, Z., Wang, J., et al. (2021). Largescale network abnormality in bipolar disorder: a multimodal meta-analysis of resting-state functional and structural magnetic resonance imaging studies. J. Affect. Disord. 292, 9-20. doi: 10.1016/j.jad.2021.05.052

Gong, J., Wang, J., Qiu, S., Chen, P., Luo, Z., Wang, J., et al. (2020). Common and distinct patterns of intrinsic brain activity alterations in major depression and bipolar disorder: voxel-based meta-analysis. Transl. Psychiatry 10:353. doi: 10.1038/s41398-020-01036-5

Grande, I., Berk, M., Birmaher, B., and Vieta, E. (2016). Bipolar disorder. Lancet 387, 1561-1572. doi: 10.1016/S0140-6736(15)00241-X

Harrison, P. J., Hall, N., Mould, A., Al-Juffali, N., and Tunbridge, E. M. (2021). Cellular calcium in bipolar disorder: systematic review and meta-analysis. Mol. Psychiatry 26, 4106-4116. doi: 10.1038/s41380-019-0622-y

Hibar, D. P., Westlye, L. T., Doan, N. T., Jahanshad, N., Cheung, J. W., Ching, C. R. K., et al. (2018). Cortical abnormalities in bipolar disorder: an MRI analysis of 6503 individuals from the ENIGMA bipolar disorder working group. Mol. Psychiatry 23, 932-942. doi: 10.1038/mp.2017.73

Hiser, J., and Koenigs, M. (2018). The multifaceted role of the ventromedial prefrontal cortex in emotion, decision making, social cognition and psychopathology. Biol. Psychiatry 83, 638-647. doi: 10.1016/j.biopsych.2017.10. 030

Keilp, J. G., Madden, S. P., Marver, J. E., Frawley, A., Burke, A. K., Herzallah, M. M., et al. (2021). Effects of ketamine Versus midazolam on neurocognition at 24 hours in depressed patients with suicidal ideation. J. Clin. Psychiatry 82:21m13921. doi: 10.4088/JCP.21 13921

Kempton, M. J., Geddes, J. R., Ettinger, U., Williams, S. C., and Grasby, P. M. (2008). Meta-analysis, database and meta-regression of 98 structural imaging studies in bipolar disorder. Arch. Gen. Psychiatry 65, 1017-1032. doi: 10.1001/archpsyc.65.9.1017

Keramatian, K., Chakrabarty, T., Saraf, G., Pinto, J. V., and Yatham, L. N. (2021). Grey matter abnormalities in first-episode mania: A systematic review and meta-analysis of voxel-based morphometry studies. Bipolar Disord. 23, 228-240. doi: 10.1111/bdi.12995

Kim, J. W., and Monteggia, L. M. (2020). Increasing doses of ketamine curtail antidepressant responses and suppress associated synaptic signaling pathways. Behav. Brain Res. 380:112378. doi: 10.1016/j.bbr.2019.112378

Liu, W., Xue, X., Xia, J., Liu, J., and Qi, Z. (2018). Swimming exercise reverses CUMS-induced changes in depression-like behaviors and hippocampal plasticity-related proteins. J. Affect. Disord. 227, 126-135. doi: 10.1016/j.jad. 2017.10.019

Lyoo, I. K., Sung, Y. H., Dager, S. R., Friedman, S. D., Lee, J. Y., Kim, S. J., et al. (2006). Regional cerebral cortical thinning in bipolar disorder. Bipolar Disord. 8, 65-74. doi: 10.1111/j.1399-5618.2006.00284.x

Magalhães, E. J. M., Sarin, L. M., Del Sant, L. C., Lucchese, A. C., Nakahira, C., Tuena, M. A., et al. (2021). A clinical rationale for assessing the impact of childhood sexual abuse on adjunctive subcutaneous esketamine for treatmentresistant depression. Front. Psychiatry 12:608499. doi: 10.3389/fpsyt.2021. 608499

Matsuo, K., Harada, K., Fujita, Y., Okamoto, Y., Ota, M., Narita, H., et al. (2019). Distinctive neuroanatomical substrates for depression in bipolar disorder versus major depressive disorder. Cereb. Cortex 29, 202-214. doi: 10.1093/cercor/bhx319

McIntyre, R. S., Berk, M., Brietzke, E., Goldstein, B. I., López-Jaramillo, C., Kessing, L. V., et al. (2020). Bipolar disorders. Lancet 396, 1841-1856. doi: 10.1016/S0140-6736(20)31544-0

Meshkat, S., Rodrigues, N. B., Di Vincenzo, J. D., Ceban, F., Jaberi, S., McIntyre, R. S., et al. (2021). Pharmacogenomics of ketamine: a systematic review. J. Psychiatr. Res. 145, 27-34. doi: 10.1016/j.jpsychires.2021.11.036

Mondimore, F. M. (2020). Bipolar Disorder: A Guide for You and Your Loved Ones (A Johns Hopkins Press Health Book). Baltimore, MD: Johns Hopkins University Press.
Muscat, S. A., Hartelius, G., Crouch, C. R., and Morin, K. W. (2021). An integrative approach to ketamine therapy may enhance multiple dimensions of efficacy: improving therapeutic outcomes with treatment resistant depression. Front. Psychiatry 12:710338. doi: 10.3389/fpsyt.2021.710338

Nikayin, S., and Sanacora, G. (2021). Evaluating the role of ketamine/esketamine in the management of major depressive disorder with suicide risk. CNS Drugs 35, 1069-1079. doi: 10.1007/s40263-021-00851-8

Nortje, G., Stein, D. J., Radua, J., Mataix-Cols, D., and Horn, N. (2013). Systematic review and voxel-based meta-analysis of diffusion tensor imaging studies in bipolar disorder. J. Affect. Disord. 150, 192-200. doi: 10.1016/j.jad.2013.05.034

Robinson, L. J., and Ferrier, I. N. (2006). Evolution of cognitive impairment in bipolar disorder: a systematic review of cross-sectional evidence. Bipolar Disord. 8, 103-116. doi: 10.1111/j.1399-5618.2006.00277.x

Schneider, M. R., DelBello, M. P., McNamara, R. K., Strakowski, S. M., and Adler, C. M. (2012). Neuroprogression in bipolar disorder. Bipolar Disord. 14, 356-374. doi: 10.1111/j.1399-5618.2012.01024.x

Sharma, A. N., Fries, G. R., Galvez, J. F., Valvassori, S. S., Soares, J. C., Carvalho, A. F., et al. (2016). Modeling mania in preclinical settings: a comprehensive review. Prog. Neuropsychopharmacol. Biol. Psychiatry 66, 22-34. doi: 10.1016/j.pnpbp.2015.11.001

Strakowski, S. M., DelBello, M. P., Zimmerman, M. E., Getz, G. E., Mills, N. P., Ret, J., et al. (2002). Ventricular and periventricular structural volumes in firstversus multiple-episode bipolar disorder. Am. J. Psychiatry 159, 1841-1847. doi: 10.1176/appi.ajp.159.11.1841

Vai, B., Bertocchi, C., and Benedetti, F. (2019). Cortico-limbic connectivity as a possible biomarker for bipolar disorder: where are we now? Expert Rev. Neurother. 19, 159-172. doi: 10.1080/14737175.2019. 1562338

Valvassori, S. S., Tonin, P. T., Dal-Pont, G. C., Varela, R. B., Cararo, J. H., Garcia, A. F., et al. (2019). Coadministration of lithium and celecoxib reverses manic-like behavior and decreases oxidative stress in a dopaminergic model of mania induced in rats. Transl. Psychiatry. 9:297. doi: 10.1038/s41398-0190637-9

Wilkowska, A., Wiglusz, M. S., Gauszko-Wegielnik, M., Wodarczyk, A., and Cubaa, W. J. (2021). Antianhedonic effect of repeated ketamine infusions in patients with treatment resistant depression. Front. Psychiatry 12:704330. doi: $10.3389 /$ fpsyt.2021.704330

Xiao, Q., Cui, D., Jiao, Q., Zhong, Y., Cao, W., Lu, G., et al. (2019). Altered regional homogeneity in pediatric bipolar disorder during manic and euthymic state: a resting-state fMRI study. Brain Imaging Behav. 13, 1789-1798. doi: 10.1007/s11682-019-00117-4

Zhou, Y., Fan, L., Qiu, C., and Jiang, T. (2015). Prefrontal cortex and the dysconnectivity hypothesis of schizophrenia. Neurosci. Bull. 31, 207-219. doi: $10.1007 / \mathrm{s} 12264-014-1502-8$

Conflict of Interest: The authors declare that the research was conducted in the absence of any commercial or financial relationships that could be construed as a potential conflict of interest.

Publisher's Note: All claims expressed in this article are solely those of the authors and do not necessarily represent those of their affiliated organizations, or those of the publisher, the editors and the reviewers. Any product that may be evaluated in this article, or claim that may be made by its manufacturer, is not guaranteed or endorsed by the publisher.

Copyright $\odot 2022$ Chen, Chen, Tian, Dou, Fang, Cai, Cheng, Chen, Chen, Ping, Lin, Chen, Zhu, Zhao, Liu, Yue, Song and Zhuo. This is an open-access article distributed under the terms of the Creative Commons Attribution License (CC BY). The use, distribution or reproduction in other forums is permitted, provided the original author(s) and the copyright owner(s) are credited and that the original publication in this journal is cited, in accordance with accepted academic practice. No use, distribution or reproduction is permitted which does not comply with these terms. 